\title{
Search for time correlation between gamma-ray bursts and data from the gravitational wave antenna EXPLORER
}

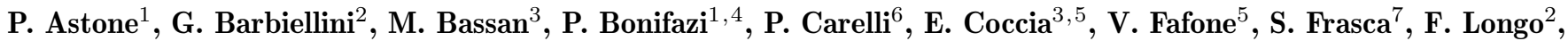 \\ E. Mauceli ${ }^{5}$, Y. Minenkov ${ }^{3}$, I. Modena ${ }^{3,5}$, G. Modestino ${ }^{5}$, A. Moleti ${ }^{3}$, G.V. Pallottino ${ }^{1,7}$, G. Pizzella ${ }^{3,5}$, \\ R. Terenzi ${ }^{1,4}$, and M. Visco ${ }^{1,4}$ \\ ${ }^{1}$ Istituto Nazionale di Fisica Nucleare (INFN), Roma, Italy \\ 2 University of Trieste and INFN, Trieste, Italy \\ 3 University of Roma "Tor Vergata" and INFN, Roma, Italy \\ 4 Istituto di Fisica dello Spazio Interplanetario, CNR, Roma, Italy \\ ${ }^{5}$ Istituto Nazionale di Fisica Nucleare, Frascati, Italy \\ 6 University of L'Aquila and INFN, L'Aquila, Italy \\ 7 University of Roma "La Sapienza", Roma, Italy
}

Received December 18, 1998; accepted May 28, 1999

\begin{abstract}
An analysis of the time correlation between Gamma-Ray Bursts from BATSE detector and EXPLORER Gravitational Wave Antenna data was performed from april 1991 to december 1996. A time interval of \pm 5 minutes around the trigger time of Gamma-Ray Bursts (GRBs) was explored. No significant excess of coincidences between GRBs and EXPLORER events with an energy of about $100 \mathrm{mK}$ was found. This result allows to exclude correlations with $1 \mathrm{~ms}$ Gravitational Wave (GW) bursts with amplitude: $h \geq 2.510^{-18}$.
\end{abstract}

Key words: gravitational waves — gamma-ray: bursts

\section{Introduction}

The phenomenon of the occurrence of powerful bursts of gamma rays lasting several seconds is still lacking an explanation. The recent discovery of an X-ray afterglow for several bursts by the BeppoSAX satellite (Costa et al. 1997) has led to the detection of high redshift absorbtion lines in the optical afterglow of some of them (Kulkarni et al. 1998), giving a clear indication of the cosmological distance of these objects. A plausible model for the source of a cosmological GRB is the coalescence of compact binary systems (Piran 1999). If so, GRBs should be associated with an emission of gravitational waves. Possible scenarios have been conceived, most of them suggesting GW fluxes below the sensitivity of presently operating GW detectors. If the source is

Send offprint requests to: F. Longo: longof@ts.infn.it assumed to be at a distance of $1 \mathrm{Gpc}$, the GW burst associated with the total conversion into $\mathrm{GWs}$ of $1 M_{\odot}$, has an amplitude of the order of $h \approx 310^{-22}$, while the present sensitivity of the best operating GW antennas with $\mathrm{SNR}=1$ is $h \approx 410^{-19}$ (Modestino et al. 1998). However due to the complete novelty of this phenomenon we have considered it worthwhile to explore whether a correlation between GRBs and data collected with the GW antenna EXPLORER (Astone et al. 1993) exists.

\section{The data}

The EXPLORER data cover certain period during the year 1991, '95, '97, because maintenance and upgrades in the experimental apparatus, for a total of about 700 non continuous days. The signals coming from the antenna are sampled with a frequency of nearly $3 \mathrm{~Hz}$ and filtered with a Wiener-Kolmogoroff filter, matched to delta-like $1 \mathrm{~ms}$ signals for maximizing the signal to noise ratio (SNR). Due to different antenna behaviour, different event selection criteria were adopted. During the 1991 we have used a fixed lower threshold in energy, while during the subsequent years this threshold was chosen in an adaptive mode: we have considered an event when the SNR was $\geq 6$. For the whole period we selected only those events with an energy of $\leq 10 \mathrm{~K}$, in order to not consider measurements presumably due to noise.

The data from BATSE detector are available in the 4B and Current Catalogs (Paciesas et al. 1998; Meegan et al. 1999). For our analysis we have considered the trigger times, peak fluxes and position informations. 
Table 1. GRBs with an event in \pm 1 s near their trigger time

\begin{tabular}{lll}
\hline GRB & peak flux & $\sin ^{4} \theta$ \\
\hline 910721 & 1.82 & 0.78 \\
$960229 \mathrm{~b}$ & 0.43 & 0.13 \\
960522 & - & 0.95 \\
$960623 \mathrm{c}$ & 0.29 & 0.98 \\
\hline
\end{tabular}

\section{Searching for coincidences}

In order to find a time correlation between the two different phenomena we have chosen as reference time $\left(t_{\mathrm{R}}\right)$ the GRB trigger time. Then we searched how many EXPLORER events occured in a time window of $\pm 1 \mathrm{~s}$ around the $t_{\mathrm{R}}$. In this way we found $4 \mathrm{GRBs}$, over the 557 analysed, that have a GW event in this time window.

The Table 1 reports the occurrence date and the peak flux counts in photons $\mathrm{cm}^{-2} \mathrm{~s}^{-1}$ of the GRBs. As the EXPLORER detector has $\sin ^{4} \theta$ dependent GW cross section, where $\theta$ is the angle between the direction of the source and the principle axis of the antenna, we also report this value in the table. These coincidence events are then analysed to search particular deviations from the $\log N-\log C$ BATSE distribution and from the $\sin ^{4} \theta$ distribution of all 557 GRBs, because we would expect an excess of events in coincidence with GRBs located closer to us and in better direction respect to the antenna.

The Kolmogorov-Smirnov test between the two distribution with the $\sin ^{4} \theta$ and the peak flux of these 4 bursts, and those obtained with the all $557 \mathrm{GRBs}$, gives respectively a value of $75 \%$ and $53 \%$ that they are compatible.

\section{Noise evaluation}

If the GRBs are located at a cosmological distance, the associated GW signal has to be very small. Because of this, in the next step of the analysis we have selected only those events that have an energy of $\leq 200 \mathrm{mK}$. So a list of 87177 GW candidate events with average energy of $100 \mathrm{mK}$ has been obtained, distributed over 652 non continuous days, with a mean rate of $\sim 133$ events per day. The time distribution of these events relative to the trigger time of each GRB in a window of $\pm 300 \mathrm{~s}$ is shown in Fig. 1 .

The total number of $393 \mathrm{GW}$ candidate events found in the $\pm 300 \mathrm{~s}$ window, over the 441 GRBs analysed, indicates that the average density of the GW events

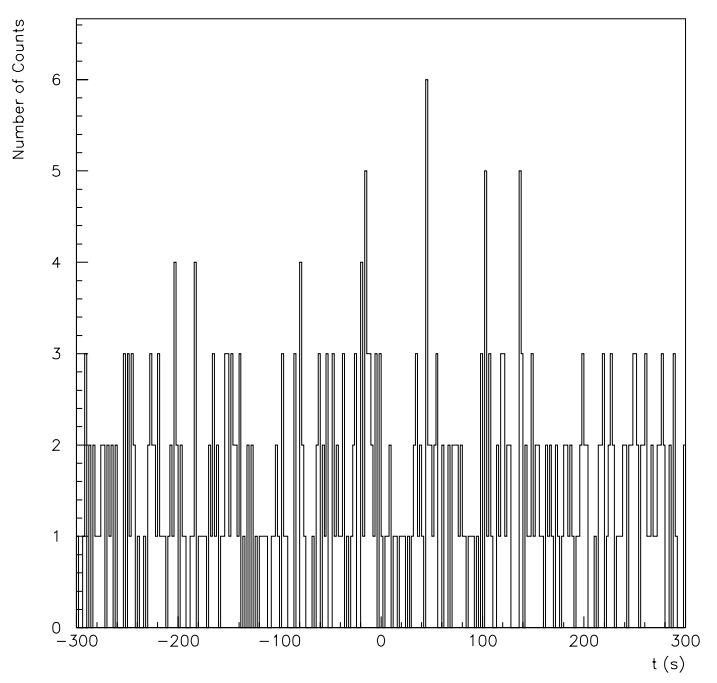

Fig. 1. Number of events with energy $\sim 100 \mathrm{mK}$ with respect to the GRB trigger time

around the trigger time, 128 events per day, is compatible with the mean rate of $\sim 133$ over the entire period. Further we notice that in the chosen window there are not significant coincidence excesses either at the trigger time or at different instants.

\section{Conclusions}

In this work we have searched for evidence of a gravitational wave signal correlating data from EXPLORER and BATSE detectors. The events from EXPLORER GW detector have an average energy $\sim 100 \mathrm{mK}$. It corresponds to an average strain amplitude of $h=2.510^{-18}$. At this sensitivity, no significant correlation is found over the considered period.

\section{References}

Astone P., et al., 1993, Phys. Rev. D 47, 362

Costa E., et al., 1997, Nat 387, 783

Kulkarni S., et al., 1998, Nat 393, 35

Modestino G., Pizzella G. and ROG collaboration, 1998, Proceedings of Vulcano '98 (in press)

Paciesas W.S., et al., 1998, http://www.batse.msfc.nasa.gov/batse/grb/catalog/4b/

Meegan C.A., et al., 1999, http://www.batse.msfc.nasa.gov/data/grb/catalog

Piran T., 1999, Physics Reports 314, 575 